\title{
Prognostic significance of using solid versus whole tumor size on high-resolution computed tomography for predicting pathologic malignant grade of tumors in clinical stage IA lung adenocarcinoma: A multicenter study
}

\author{
Yasuhiro Tsutani, MD, PhD, ${ }^{\text {a }}$ Yoshihiro Miyata, $\mathrm{MD}, \mathrm{PhD},{ }^{\text {a }}$ Haruhiko Nakayama, $\mathrm{MD}, \mathrm{PhD},{ }^{\mathrm{b}}$ \\ Sakae Okumura, MD, PhD, ${ }^{\mathrm{c}}$ Shuji Adachi, MD, PhD, ${ }^{\mathrm{d}}$ Masahiro Yoshimura, $\mathrm{MD}, \mathrm{PhD},{ }^{\mathrm{e}}$ and \\ Morihito Okada, MD, $\mathrm{PhD}^{\mathrm{a}}$
}

\begin{abstract}
Objectives: The present multicenter study compared the usefulness of the solid tumor size with that of the whole tumor size on preoperative high-resolution computed tomography for predicting pathologic high-grade malignancy (positive lymphatic, vascular, or pleural invasion) and the prognosis of clinical stage IA lung adenocarcinoma.

Methods: We performed high-resolution computed tomography and F-18 fluorodeoxyglucose-positron emission tomography/computed tomography before curative surgical resection in 502 patients with clinical stage IA lung adenocarcinoma. The revised maximum standardized uptake values on F-18 fluorodeoxyglucose-positron emission tomography/computed tomography were used to correct interinstitutional discrepancies. The whole and solid tumor sizes on high-resolution computed tomography were then analyzed in relation to surgical results.
\end{abstract}

\begin{abstract}
Results: The mean whole and solid tumor size was $1.97 \pm 0.59 \mathrm{~cm}$ and $1.20 \pm 0.88 \mathrm{~cm}$, respectively. The receiver operating characteristics area under the curve for the whole and solid tumor sizes used to identify highgrade malignancy were 0.590 and 0.829 , respectively. Multiple logistic regression analyses demonstrated solid tumor size $(P<.001)$ and maximum standardized uptake values of the tumor $(P<.001)$ as independent variables for the prediction of high-grade malignancy. Multivariate Cox analysis of disease-free survival demonstrated the former (hazard ratio, 2.30; 95\% confidence interval, 1.46-3.63; $P<.001$ ) and latter (hazard ratio, 1.08; 95\% confidence interval, $1.00-1.17 ; P=.05$ ) as independent prognostic factors.
\end{abstract}

Conclusions: The solid tumor size on high-resolution computed tomography and maximum standardized uptake values on positron emission tomography/computed tomography have greater predictive value for high-grade malignancy and prognosis in clinical stage IA lung adenocarcinoma than that of whole tumor size. (J Thorac Cardiovasc Surg 2012;143:607-12)

Recent advances in high-resolution computed tomography (HRCT) and the widespread application of computed tomography (CT) screening have enhanced the discovery of small lung cancers, particularly adenocarcinoma. ${ }^{1,2}$ These often contain a nonsolid component that presents as ground glass opacity (GGO) on HRCT. $^{2,3}$ Several investigators have reported that GGO is closely associated with bronchioloalveolar carcinoma. ${ }^{3,4}$ Noguchi and

\footnotetext{
From the Department of Surgical Oncology, ${ }^{a}$ Hiroshima University, Hiroshima, Japan; Department of Thoracic Surgery, ${ }^{b}$ Kanagawa Cancer Center, Yokohama, Japan; Department of Thoracic Surgery, ${ }^{c}$ Cancer Institute Hospital, Tokyo, Japan; and Departments of Radiology ${ }^{\mathrm{d}}$ and Thoracic Surgery, ${ }^{\mathrm{e}}$ Hyogo Cancer Center Akashi, Japan.

Disclosures: Authors have nothing to disclose with regard to commercial support.

Received for publication June 30, 2011; revisions received Sept 7, 2011; accepted for publication Oct 20, 2011; available ahead of print Nov 21, 2011.

Address for reprints: Morihito Okada, MD, PhD, Department of Surgical Oncology, Research Institute for Radiation Biology and Medicine, Hiroshima University, 1-2-3 Kasumi, Minami-ku, Hiroshima 734-0037, Japan (E-mail: morihito@ hiroshima-u.ac.jp).

$0022-5223 / \$ 36.00$

Copyright (C) 2012 by The American Association for Thoracic Surgery

doi:10.1016/j.jtcvs.2011.10.037
}

colleagues ${ }^{5}$ reported that type A and B small peripheral adenocarcinomas (localized bronchioloalveolar carcinoma without foci of active fibroblastic proliferation) demonstrated no lymph node metastasis and a favorable prognosis (100\% 5-year survival rate). We therefore hypothesized that the GGO component is not related to malignancy and prognosis, implying that only the solid component of the tumor on HRCT (solid tumor size) is indicative of malignancy and prognosis.

The purpose of the present multicenter study was to evaluate and compare the usefulness of the solid tumor size with that of the whole tumor size as seen on preoperative HRCT for determining pathologic high-grade malignancy (positive for lymphatic [LI], vascular [VI], or pleural invasion $[\mathrm{PI}])$ and the prognosis in cases of clinical stage IA lung adenocarcinoma.

\section{PATIENTS AND METHODS \\ Patients}

We enrolled 502 patients with clinical T1N0M0 stage IA lung adenocarcinoma at 4 institutions (Hiroshima University, Kanagawa Cancer Center, Cancer Institute Hospital, and Hyogo Cancer Center) from August 1, 2005 


$$
\begin{aligned}
& \text { Abbreviations and Acronyms } \\
& \begin{aligned}
\text { CI } & =\text { confidence interval } \\
\text { CT } & =\text { computed tomography } \\
\text { DFS } & =\text { disease-free survival } \\
\text { FDG- } & =\text { F-18 fluorodeoxyglucose-positron } \\
\text { PET } & \text { emission tomography } \\
\text { GGO } & =\text { ground glass opacity } \\
\text { HR } & =\text { hazard ratio } \\
\text { HRCT } & =\text { high-resolution computed tomography } \\
\text { LI } & =\text { lymphatic invasion } \\
\text { PI } & =\text { pleural invasion } \\
\text { SUV } & =\text { standardized uptake value } \\
\text { VI } & =\text { vascular invasion }
\end{aligned}
\end{aligned}
$$

to December 31, 2009. HRCT and F-18 fluorodeoxyglucose-positron emission tomography/CT (FDG-PET/CT) followed by curative R0 resection were performed for all patients, who underwent staging according to the 7th edition of the TNM classification of malignant tumors. ${ }^{6}$ Sublobar resections (segmentectomy or wedge resection) were allowed if the tumor mainly consisted of GGO or had no lymph node metastasis through intraoperative assessment. The institutional review board of each institution provided appropriate approval for the present multicenter study. They waived the requirement of informed consent from the individual patients for the present retrospective review of the prospective database. The data were compiled in a Microsoft Excel 2010 file (Microsoft, Redmond, WA).

\section{HRCT Scanning}

Chest images were obtained using 16-row, multidetector CT, independent of the subsequent FDG-PET/CT examinations. High-resolution images of the tumors were acquired using the following parameters: 120 $\mathrm{kVp}, 200 \mathrm{~mA}, 1$ - to 2 -mm section thickness, $512 \times 512$ pixel resolution, scanning time 0.5 to $1 \mathrm{sec}$, and a high spatial reconstruction algorithm with a $20-\mathrm{cm}$ field of view and mediastinal (level, $40 \mathrm{HU}$; width, 400 $\mathrm{HU}$ ) and lung (level, $-600 \mathrm{HU}$; width, $1600 \mathrm{HU}$ ) window settings. GGO was defined as a misty increase in lung attenuation that did not obscure the underlying vascular markings. We defined the solid tumor size as the maximum dimension of the solid component of the lung windows excluding GGO (Figure 1).

\section{FDG-PET/CT Scanning}

The patients were instructed to fast for more than 4 hours before intravenous injection of 74 to $370 \mathrm{MBq}$ FDG and then made to relax for at least 1 hour before FDG-PET/CT scanning. Blood glucose was calculated before the tracer injection to confirm a level of less than $150 \mathrm{mg} / \mathrm{dL} .{ }^{7}$ Patients with blood glucose values of $150 \mathrm{mg} / \mathrm{dL}$ or greater were excluded from PET/CT acquisition. The images were obtained using Discovery ST (GE Healthcare, Little Chalfont, UK), Aquiduo (Toshiba Medical Systems, Tochigi, Japan), or Biograph Sensation16 (Siemens Healthcare, Erlangen, Germany) integrated PET/CT scanners. Low-dose, unenhanced CT images of 2- to 4-mm section thickness for attenuation correction and localization of lesions identified by PET were obtained from the head to the pelvic floor of each patient using a standard protocol. Immediately after CT, PET covered the identical axial field of view for 2 to 4 minutes per table position, depending on the condition of the patient and scanner performance. All PET images with a 50-cm field of view were reconstructed using an iterative algorithm with CT-derived attenuation correction. Variations in the standardized uptake values (SUVs) among institutions were minimized using an anthropomorphic body phantom. A calibration factor was analyzed by dividing the actual SUV by the gauged mean SUV in the phantom background to decrease interinstitutional SUV inconsistencies. The final SUV reported in the present study is referred to as the revised maximum SUV (maxSUV) ${ }^{8,9}$ The adjustment of the interinstitutional variability in the SUV narrowed the range from 0.89 to 1.24 to 0.97 to 1.18 when the maxSUV ratio was expressed as the maxSUV of each institute relative to the maxSUV of the control institute.

\section{Statistical Analysis}

The data are presented as the numbers and percentages or mean \pm standard deviation, unless otherwise stated. Pathologic high-grade malignancy was defined as positive LI, VI, or PI. The receiver operating characteristic curves of the whole and solid tumor sizes were used for the prediction of LI, VI, or PI, high-grade malignancy (LI, VI, or PI), and lymph node metastasis. We also performed multiple logistic regression analyses to determine the independent variables related to whole tumor size, solid tumor size, and maxSUV for the prediction of the pathologic finding of high-grade malignancy. Disease-free survival (DFS) was defined as interval from the date of surgery until the first event (relapse or death from any cause) or the last follow-up visit. The duration of DFS was analyzed using the Kaplan-Meier method. Differences in DFS were assessed using the log-rank test. To assess the potential independent effects of clinical tumor size on DFS, we performed multivariate analyses with the Cox proportional hazards model using variables with $P<.05$ on the univariate analyses; $P<.05$ was considered statistically significant. The data were statistically analyzed using the Statistical Package for Social Sciences software, version 10.5 (SPSS, Chicago, 11).

\section{RESULTS}

The characteristics of the 502 study patients are summarized in Table 1. The mean follow-up period after surgery was $19.8 \pm 12.2$ months, during which disease recurred in 29 patients $(5.8 \%)$. Of the 29 recurrence cases, $9(1.8 \%)$ were local, including mediastinal lymph node metastasis, $3(0.6 \%)$ were local and distant, and $17(3.4 \%)$ were distant. The mean whole tumor and solid tumor size on HRCT was $1.97 \pm 0.59 \mathrm{~cm}$ and $1.20 \pm 0.88 \mathrm{~cm}$, respectively. LI, VI, and PI was evident in $76(15.1 \%), 92$ $(18.3 \%)$, and $56(11.2 \%)$ patients, respectively, and the lymph nodes were involved in $38(7.8 \%)$.

The receiver operating characteristic area under the curve values of the whole and solid tumor sizes used for predicting LI, VI, PI, pathologic high-grade malignancy (LI, VI, or PI), and lymph node metastasis are given in Table 2 and Figure 2. The predictability of all outcomes on the basis of solid tumor size was better than that using the whole tumor size for all subjects.

Although a significant relationship was noted between the whole tumor size and maxSUV (decision coefficient, $\left.r^{2}=0.015 ; P=.007\right)$, a much more significant relationship was detected between the solid tumor size and maxSUV $\left(r^{2}=0.211, P<.001\right)$.

The possible predictors of pathologic high-grade malignancy (LI, VI, or PI) were also investigated (Table 3). Although whole tumor size, solid tumor size, and maxSUV were all significant determinants on the univariate analyses, the solid tumor size and maxSUV were significant determinants on multivariate analysis. Whole tumor size was not an independent predictor of high-grade malignancy. 


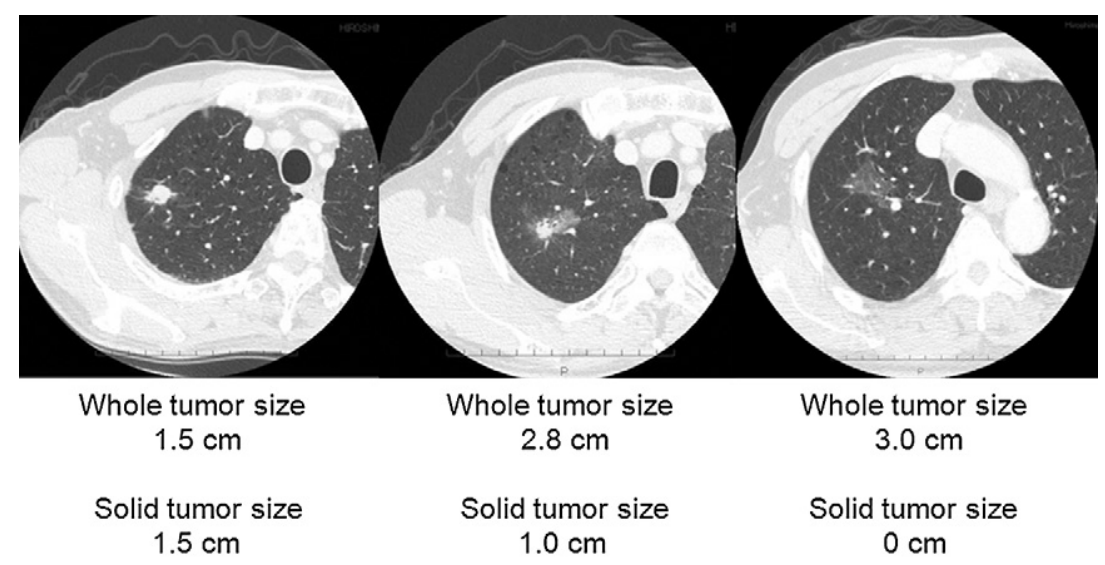

A

B

C

FIGURE 1. Examples of whole and solid tumor sizes on high-resolution computed tomography. A, Whole tumor size, $1.5 \mathrm{~cm}$ and solid tumor size, $1.5 \mathrm{~cm}$. $\mathrm{B}$, Whole tumor size, $2.8 \mathrm{~cm}$ and solid tumor size, $1.0 \mathrm{~cm}$. C, Whole tumor size, $3.0 \mathrm{~cm}$ and solid tumor size, $0 \mathrm{~cm}$.

No significant difference in DFS was identified between patients with a whole tumor size of $2.0 \mathrm{~cm}$ or less $(\mathrm{n}=289$; 3-year DFS rate, $92.5 \%$ ) and those with a whole tumor size greater than $2.0 \mathrm{~cm}(\mathrm{n}=213$; 3-year DFS rate, $86.7 \%$; $P=.511 ;$ Figure $3, A)$. In contrast, a significant difference in DFS was identified between patients with a solid tumor size of $2.0 \mathrm{~cm}$ or less $(\mathrm{n}=400 ; 3$-year DFS rate, $93.2 \%)$ and those with a solid tumor size greater than $2.0 \mathrm{~cm}$ $(\mathrm{n}=102$; 3-year DFS rate, 78.9\%; $P=.013$; Figure 3, $B)$.

Univariate analysis of DFS among the 502 patients with adenocarcinoma included preoperative tumor factors (eg, whole tumor size, solid tumor size, and maxSUV; Table 4) as variables. A larger solid tumor size and greater maxSUV were associated with a shorter DFS, but the whole tumor size was not associated with DFS. Moreover, the multivariate analysis that included a solid tumor size and maxSUV as variables demonstrated that solid tumor size (hazard ratio [HR], 2.30; 95\% confidence interval [CI], $1.46-3.63 ; P<.001)$ and maxSUV (HR, $1.08 ; 95 \% \mathrm{CI}$, $1.00-1.17 ; P=0.05)$ were independent prognostic factors for DFS (Table 4).

In another multivariate analysis, including the variables of gender, operation type, solid tumor size, maxSUV, and lymph node metastasis, the solid tumor size (HR, 2.00; 95\% CI, 1.19-3.36; $P=.009$ ), maxSUV (HR, 1.10; $95 \%$ CI, 1.01-1.20; $P=.034)$, and lymph node metastasis (HR, 7.17; 95\% CI, 3.18-16.2; $P<.001$ ) were independent prognostic factors for DFS. In contrast, the whole tumor size was not an independent factor for DFS. However, maxSUV and lymph node metastasis were significant in the model replacing solid tumor size with whole tumor size.

\section{DISCUSSION}

The frequency of identification of small lung cancers has increased since CT and enhanced scanning have become routine procedures. Such small tumors, especially in lung adenocarcinomas, often contain GGO components as visualized on HRCT. ${ }^{1-4,10}$ Generally, clinical physicians measure the tumor size according to the TNM classification by including the GGO components visualized on HRCT.

On the basis of our hypothesis that the solid components, and not the GGO components, of tumors as visualized on HRCT indicate malignancy and prognosis, we evaluated the role of solid tumor size (the size without the GGO component) in cases of clinical stage IA lung adenocarcinoma

TABLE 1. Patient characteristics

\begin{tabular}{lc}
\hline \multicolumn{1}{c}{ Variable } & $\mathbf{n}=\mathbf{5 0 2}$ \\
\hline Age (y) & $65.3 \pm 9.6$ \\
Gender & \\
Male & $223(44.4)$ \\
Female & $279(55.6)$ \\
Tumor size & \\
$\quad$ Whole (cm) & $1.97 \pm 0.59$ \\
Solid (cm) & $1.20 \pm 0.88$ \\
MaxSUV & $2.84 \pm 3.03$ \\
Procedure & \\
Lobectomy & $320(63.7)$ \\
Limited (wedge plus segmentectomy) & $182(36.3)$ \\
Lymphatic invasion & \\
$\quad$ Negative & $426(84.9)$ \\
Positive & $76(15.1)$ \\
Vascular invasion & \\
$\quad$ Negative & $410(81.7)$ \\
Positive & $92(18.3)$ \\
Pleural invasion & \\
$\quad$ Negative & $446(88.8)$ \\
Positive & $56(11.2)$ \\
Lymph node metastasis & \\
Negative & $464(92.4)$ \\
Positive & $38(7.6)$ \\
\hline Data in parentheses are percentages. maxSUV, Maximum standardized uptake value. \\
\end{tabular}


TABLE 2. Receiver operative characteristic area under the curve values of whole and solid tumor sizes used to predict pathologic findings

\begin{tabular}{|c|c|c|c|c|}
\hline \multirow[b]{2}{*}{ Variable } & \multicolumn{2}{|c|}{ Whole tumor size } & \multicolumn{2}{|c|}{ Solid tumor size } \\
\hline & $\operatorname{AUC}(95 \%$ CI $)$ & $P$ value & $\operatorname{AUC}(95 \%$ CI $)$ & $P$ value \\
\hline LI & $0.560(0.494-0.626)$ & .092 & $0.777(0.733-0.820)$ & $<.001$ \\
\hline VI & $0.634(0.571-0.697)$ & .001 & $0.829(0.789-0.868)$ & $<.001$ \\
\hline PI & $0.576(0.499-0.653)$ & .039 & $0.771(0.723-0.820)$ & $<.001$ \\
\hline LI or VI or PI & $0.590(0.534-0.646)$ & .002 & $0.829(0.794-0.864)$ & $<.001$ \\
\hline $\mathrm{N}$ & $0.603(0.519-0.688)$ & .034 & $0.761(0.703-0.819)$ & $<.001$ \\
\hline
\end{tabular}

AUC, Area under the curve; $C I$, confidence interval; $L I$, lymphatic invasion; $V I$, vascular invasion; $P I$, pleural invasion; $N$, lymph node metastasis.

and compared its prognostic significance with that of whole tumor size (the size including the GGO component).

All receiver operating characteristic area under the curves for predicting LI, VI, PI, high-grade malignancy, and lymph node metastasis were larger for the solid tumor size than those for the whole tumor size. Moreover, the solid tumor size and maxSUV were identified as independent predictive factors for high-grade malignancy. These findings indicate that the solid tumor size, and not whole tumor size, reflects the pathologic findings related to clinical tumor malignancy. Concerning the relationship between tumor size and lymph node metastasis, we should take account of stage migration because $36 \%$ of the patients underwent sublobar resection.

The 7th TNM classification for lung cancer divided the $\mathrm{T} 1$ descriptor into T1a (tumors $\leq 2 \mathrm{~cm}$ ) and T1b (tumors 2-3 cm) on the basis of the optimal cutoff point for 5-year survival. ${ }^{11}$ We analyzed each DFS point in stage IA adenocarcinoma according to the cutoff point of $2 \mathrm{~cm}$ using the whole and solid tumor sizes. Although no significant difference in DFS was observed between patients with a whole tumor size of $2.0 \mathrm{~cm}$ or less and those with a whole tumor size of greater than $2.0 \mathrm{~cm}$, a significant difference in DFS was identified between patients with a solid tumor
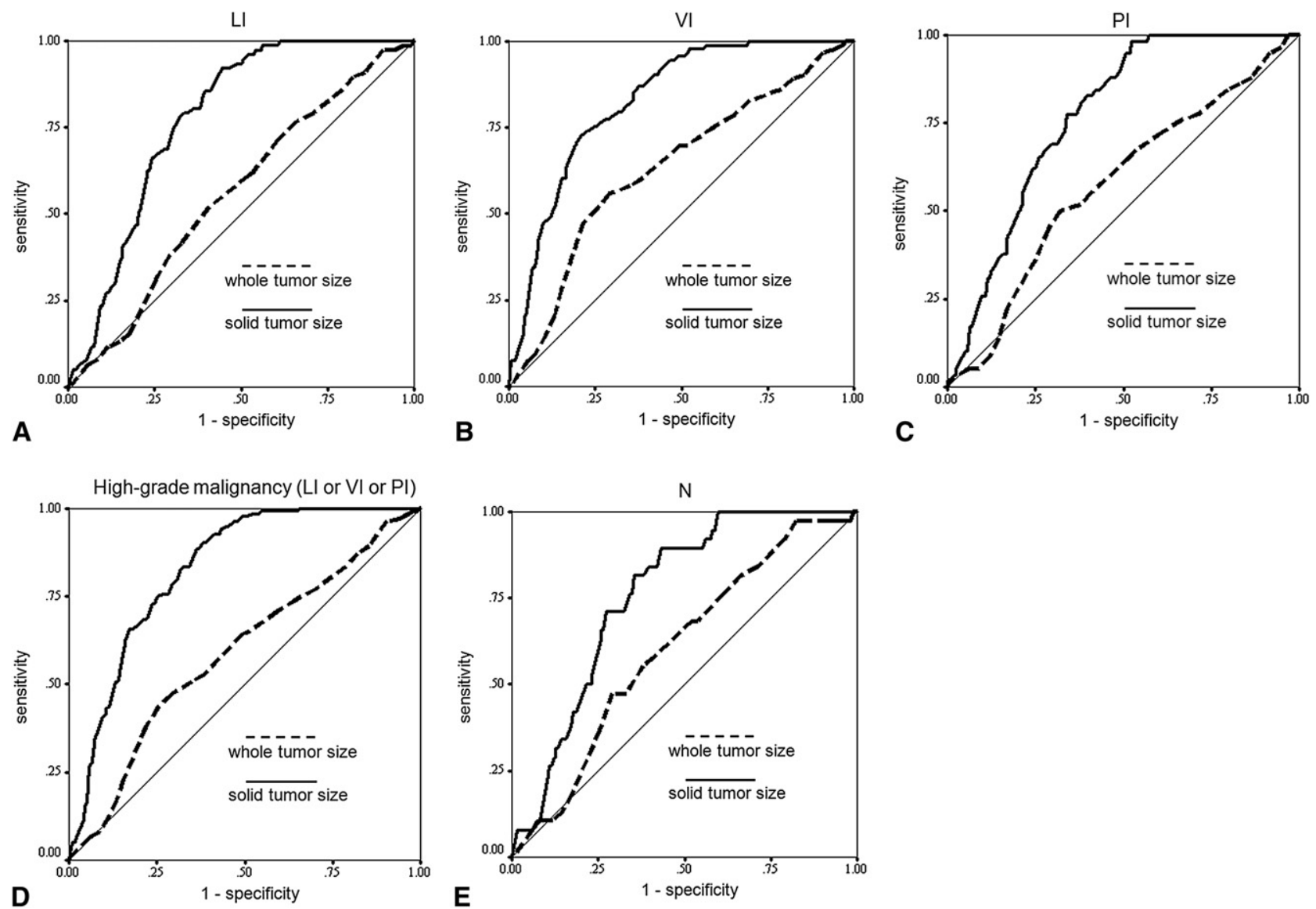

FIGURE 2. Receiver operating characteristic area under the curve for detecting (A) lymphatic invasion (LI) (B) vascular invasion (VI) (C) pleural invasion (PI) (D) high-grade malignancy (LI, VI, or PI), and (E) lymph node metastasis (N) for whole and solid tumor sizes. 
TABLE 3. Analysis of possible predictors of pathologic high-grade malignancy (positive for lymphatic, vascular, or pleural invasion)

\begin{tabular}{lcr}
\hline \multicolumn{1}{c}{ Variable } & OR $(\mathbf{9 5} \% \mathbf{C I})$ & $\boldsymbol{P}$ value \\
\hline Univariate analysis & & \\
$\quad$ Whole tumor size $(\mathrm{cm})$ & $1.70(1.21-2.39)$ & .002 \\
$\quad$ Solid tumor size $(\mathrm{cm})$ & $4.86(3.57-6.63)$ & $<.001$ \\
$\quad$ MaxSUV & $1.51(1.37-1.66)$ & $<.001$ \\
Multivariate analysis & & \\
$\quad$ Model 1 & $1.39(0.94-2.05)$ & .97 \\
$\quad$ Whole tumor size $(\mathrm{cm})$ & $1.49(1.35-1.64)$ & $<.001$ \\
$\quad$ MaxSUV & & \\
$\quad$ Model 2 & $3.77(2.69-5.27)$ & $<.001$ \\
$\quad$ Solid tumor size $(\mathrm{cm})$ & $1.25(1.14-1.37)$ & $<.001$ \\
$\quad$ MaxSUV & & \\
OR, Odds ratio; $C I$, confidence interval; $\operatorname{maxSUV}$, maximum standardized uptake \\
value.
\end{tabular}

size of $2.0 \mathrm{~cm}$ or less and those with a solid tumor size of greater than $2.0 \mathrm{~cm}$. Furthermore, multivariate Cox analysis revealed that solid tumor size and maxSUV were independent prognostic factors for DFS, and whole tumor size was not. These results have indicated that the solid tumor size more closely correlates with survival and the pathologic findings than does the whole tumor size.

The present study demonstrated that approximately one half of the patients with whole tumors greater than $2 \mathrm{~cm}$ could be restaged clinically as having a solid tumor of 2 $\mathrm{cm}$ or less. This means that even tumors greater than $2 \mathrm{~cm}$ often contain a GGO component. Currently, phase III randomized trials of standard lobectomy versus experimental limited resection for small ( $\leq 2 \mathrm{~cm}$ in diameter) peripheral nonsmall-cell lung cancers are ongoing in the United States (Cancer and Leukemia Group B trial 14053) and Japan (Japan Clinical Oncology Group trial 0802/West Japan Oncology Group trial 4607L). Among patients with lung adenocarcinomas and a whole tumor size greater than 2.0 $\mathrm{cm}, 50 \%$ have a good prognosis and could be future candidates for limited surgery such as segmentectomy and wedge resection of the lung if the surgical margin is adequate. No difference was seen in DFS among patients with a solid tumor size of $2 \mathrm{~cm}$ or less, who were treated with lobectomy versus sublobar resection in the present study (data not shown); however, this finding should be confirmed by prospective studies.

Several investigators have reported that the prognosis of patients with lung adenocarcinoma and a large GGO component visualized on HRCT was much better than that of patients with other adenocarcinoma types, irrespective of the maximal tumor dimension. ${ }^{12-15}$ In addition, a large prospective study has examined the specificity, sensitivity, and accuracy of the radiologic diagnoses of lymphatic/ vessel invasion and nodal involvement of clinical T1N0M0 adenocarcinoma made according to the HRCT findings. ${ }^{16}$ The outcomes failed to corroborate the
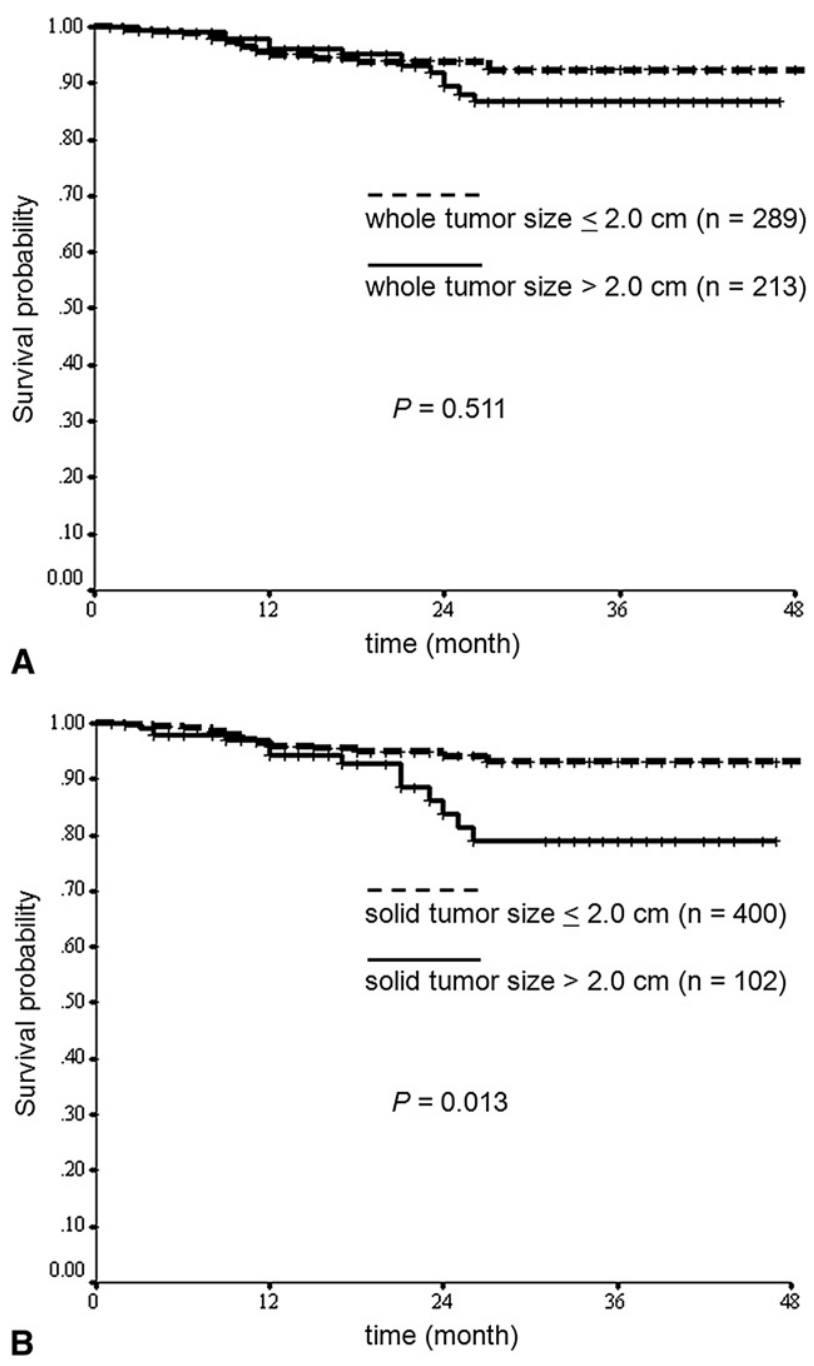

FIGURE 3. Disease-free survival (DFS) curves of patients according to tumor size on high-resolution computed tomography. A, Three-year DFS rate of $92.5 \%$ and $86.7 \%$ for a whole tumor size of $2.0 \mathrm{~cm}$ or less and greater than $2.0 \mathrm{~cm}$, respectively $(P=.511)$. B, Three-year DFS rate of $93.2 \%$ and $78.9 \%$ for solid tumor size of less than $2.0 \mathrm{~cm}$ and greater than $2.0 \mathrm{~cm}$, respectively $(P=.013)$.

predetermined criteria using the GGO ratio for specificity. This indicates that using the solid tumor size is much simpler than using the GGO ratio; furthermore, the solid tumor size can be applied to the $\mathrm{T}$ descriptor in the TNM classification.

In the present study, the maxSUV on FDG-PET/CT was also a useful factor for predicting pathologic high-grade malignancy and DFS. We have previously reported on the usefulness of maxSUV on PET/CT as a predictor of malignancy and the prognosis of adenocarcinoma of the lung but not for squamous cell carcinoma of the lung. ${ }^{8,9,17,18}$ In those reports, the maxSUV was closely related to the occurrence of LI, VI, PI, and nodal metastasis and was an independent predictive factor for DFS. ${ }^{8,9,17,18}$ The interinstitutional 
TABLE 4. Univariate and multivariate analyses of disease-free survival

\begin{tabular}{llc}
\hline \multicolumn{1}{c}{ Variable } & HR $(\mathbf{9 5} \% \mathbf{C I})$ & $\boldsymbol{P}$ value \\
\hline Univariate analysis & & \\
$\quad$ Whole tumor size $(\mathrm{cm})$ & $1.47(0.78-2.77)$ & .23 \\
Solid tumor size $(\mathrm{cm})$ & $2.49(1.60-3.89)$ & $<.001$ \\
$\quad$ MaxSUV & $1.10(1.04-1.17)$ & $<.001$ \\
Multivariate analysis & & \\
$\quad$ Solid tumor size $(\mathrm{cm})$ & $2.30(1.46-3.63)$ & $<.001$ \\
$\quad$ MaxSUV & $1.08(1.00-1.17)$ & .05 \\
\hline
\end{tabular}

$H R$, Hazard ratio; $\max S U V$, maximum standardized uptake value.

variability in the SUVs obtained in the present study was minimized using an anthropomorphic body phantom. Therefore, quantitative SUVs adjusted by phantom studies can be quite dependable, and such correction will help to overcome a major limitation concerning the wide variability in SUVs among institutions in multicenter PET studies.

Generally, FDG uptake is dependent on tumor size, ${ }^{19,20}$ and the present study demonstrated that solid tumor size was more closely related to the maxSUV than was the whole tumor size and that the solid tumor size is a potential marker of malignancy and prognosis. This finding is important for lung adenocarcinomas with a GGO component visualized on HRCT.

\section{CONCLUSIONS}

The predictive values of the solid tumor size visualized on HRCT and the maxSUV on PET/CT for pathologic high-grade malignancy and prognosis in cases of clinical stage IA lung adenocarcinoma were greater than those of whole tumor size. We recommend that the solid tumor size be used to determine the T descriptor in the TNM classification of lung cancer and should be defined as the true tumor size in cases of lung adenocarcinoma with a GGO component visualized on HRCT.

\section{References}

1. Okada M, Koike T, Higashiyama M, Yamato Y, Kodama K, Tsubota N. Radical sublobar resection for small-sized non-small cell lung cancer: a multicenter study. J Thorac Cardiovasc Surg. 2006;132:769-75.

2. Nakayama H, Yamada K, Saito H, Oshita F, Ito H, Kameda Y, et al. Sublobar resection for patients with peripheral small adenocarcinomas of the lung: surgical outcome is associated with features on computed tomographic imaging. Ann Thorac Surg. 2007;84:1675-9.

3. Nakata M, Saeki H, Takata I, Segawa Y, Mogami H, Mandai K, et al. Focal ground-glass opacity detected by low-dose helical CT. Chest. 2002;121:1464-7.

4. Jang HJ, Lee KS, Kwon OJ, Rhee CH, Shim YM, Han J. Bronchioloalveolar carcinoma: focal area of ground-glass attenuation at thin-section CT as an early sign. Radiology. 1996;199:485-8.
5. Noguchi M, Morikawa A, Kawasaki M, Matsuno Y, Yamada T, Hirohashi S, et al. Small adenocarcinoma of the lung: histologic characteristics and prognosis. Cancer. 1995;75:2844-52.

6. Goldstraw P, Crowley J, Chansky K, Giroux DJ, Groome PA, Rami-Porta R, et al., International Association for the Study of Lung Cancer International Staging Committee; Participating Institutions. The IASLC Lung Cancer Staging Project: proposals for the revision of the TNM stage groupings in the forthcoming (seventh) edition of the TNM Classification of Malignant Tumours. J Thorac Oncol. 2007;2:706-14.

7. Delbeke D, Coleman RE, Guiberteau MJ, Brown ML, Royal HD, Siegel BA, et al. Procedure guideline for tumor imaging with 18F-FDG PET/CT 1.0. J Nucl Med. 2006;47:885-95.

8. Nakayama H, Okumura S, Daisaki H, Kato Y, Uehara H, Adachi S, et al. Value of integrated positron emission tomography revised using a phantom study to evaluate malignancy grade of lung adenocarcinoma. Cancer. 2010;116:3170-7.

9. Okada M, Nakayama H, Okumura S, Daisaki H, Adachi S, Yoshimura M, et al. Multicenter analysis of high-resolution computed tomography and positron emission tomography/computed tomography findings to choose therapeutic strategies for clinical stage IA lung adenocarcinoma. J Thorac Cardiovasc Surg. 2011;141:1384-91.

10. Suzuki K, Kusumoto M, Watanabe S, Tsuchiya R, Asamura H. Radiologic classification of small adenocarcinoma of the lung: radiologic-pathologic correlation and its prognostic impact. Ann Thorac Surg. 2006;81:413-9.

11. Rami-Porta R, Ball D, Crowley J, Giroux DJ, Jett J, Travis WD, et al. The IASLC lung cancer staging project: proposals for the revision of the $\mathrm{T}$ descriptors in the forthcoming (seventh) edition of the TNM classification for lung cancer. $J$ Thorac Oncol. 2007;2:593-602.

12. Aoki T, Tomoda Y, Watanabe H, Nakata H, Kasai T, Hashimoto H, et al. Peripheral lung adenocarcinoma: correlation of thin-section CT findings with histologic prognostic factors and survival. Radiology. 2001;220:803-9.

13. Takashima S, Maruyama Y, Hasegawa M, Yamanda T, Honda T, Kadoya M, et al. Prognostic significance of high-resolution CT findings in small peripheral adenocarcinoma of the lung: a retrospective study on 64 patients. Lung Cancer. 2002; 36:289-95.

14. Suzuki K, Asamura H, Kusumoto M, Kondo H, Tsuchiya R. "Early" peripheral lung cancer: prognostic significance of ground glass opacity on thin-section computed tomographic scan. Ann Thorac Surg. 2002;74:1635-9.

15. Ohde Y, Nagai K, Yoshida J, Nishimura M, Takahashi K, Suzuki K, et al. The proportion of consolidation to ground-glass opacity on high resolution CT is a good predictor for distinguishing the population of non-invasive peripheral adenocarcinoma. Lung Cancer. 2003;42:303-10.

16. Suzuki K, Koike T, Asakawa T, Kusumoto M, Asamura H, Nagai K, et al. A prospective radiological study of thin-section computed tomography to predict pathological noninvasiveness in peripheral clinical IA lung cancer (Japan Clinical Oncology Group 0201). J Thorac Oncol. 2011;6:751-6.

17. Okada M, Tauchi S, Iwanaga K, Mimura T, Kitaura Y, Watanabe H, et al. Associations among bronchioloalveolar carcinoma components, positron emission tomographic findings, and malignant behavior in small lung adenocarcinomas. J Thorac Cardiovasc Surg. 2007;133:1448-54.

18. Tsutani Y, Miyata Y, Misumi K, Ikeda T, Mimura T, Hihara J, et al. Difference in prognostic significance of maximum standardized uptake value on [18F]-fluoro-2-deoxyglucose positron emission tomography between adenocarcinoma and squamous cell carcinoma of the lung. Jpn J Clin Oncol. 2011;41:890-6.

19. Vansteenkiste JF, Stroobants SG, Dupont PJ, De Leyn PR, Verbeken EK, Deneffe GJ, et al. Prognostic importance of the standardized uptake value on (18)F-fluoro-2-deoxy-glucose-positron emission tomography scan in nonsmall-cell lung cancer: an analysis of 125 cases. Leuven Lung Cancer Group. J Clin Oncol. 1999;17:3201-6.

20. Downey RJ, Akhurst T, Gonen M, Vincent A, Bains MS, Larson S, et al. Preoperative F-18 fluorodeoxyglucose-positron emission tomography maximal standardized uptake value predicts survival after lung cancer resection. J Clin Oncol. 2004;22:3255-60. 